\title{
Ab-Initio Calculations for Solvus Temperatures of Pd-Rich PdRu Alloys: Real-Space Cluster Expansion and Cluster Variation Method
}

\author{
Chang Liu ${ }^{1}$, Mitsuhiro Asato ${ }^{2, *}$, Nobuhisa Fujima ${ }^{3}$, Toshiharu Hoshino ${ }^{4}$, Ying Chen ${ }^{5}$ and Tetsuo Mohri ${ }^{6}$ \\ ${ }^{1}$ National Institute for Materials Science, Tsukuba 305-0047, Japan \\ ${ }^{2}$ National Institute of Technology, Niihama College, Niihama 792-8580, Japan \\ ${ }^{3}$ Graduate School of Engineering, Shizuoka University, Hamamatsu 432-8561, Japan \\ ${ }^{4}$ Faculty of Engineering, Shizuoka University, Hamamatsu 432-8561, Japan \\ ${ }^{5}$ Graduate School of Engineering, Tohoku University, Sendai 980-8579, Japan \\ ${ }^{6}$ Institute for Materials Research, Tohoku University, Sendai 980-8577, Japan
}

\begin{abstract}
We present the ab-initio calculations for the solvus temperatures $\left(T_{\text {solvus }}\right)$ of $\operatorname{Pd}_{1-c} \operatorname{Ru}_{c}(0.03 \leq \mathrm{c} \leq 0.12)$, in which the interaction energies (IEs) among the Ru impurities in Pd are determined by the full-potential Korringa-Kohn-Rostoker Green's function method, combined with the generalized gradient approximation in the density functional theory. The configurational entropy calculations are based on the cluster variation method (CVM) within the tetrahedron approximation. In order to take into account the long-range 2-body IEs, we renormalized the 1st-nearest neighbor $(n n)$ IE by including the $2 \sim 10$ th- $n n$ IEs. We also take into account the following three effects on the IEs among the Ru impurities; (1) the local lattice distortion for the 1st- $n n$ IE, existing even at $T=0 \mathrm{~K}$, (2) the lattice thermal vibration by the Debye-Grüneisen model, and (3) the $T$-dependence of the electron excitation due to the Fermi-Dirac distribution. The calculated results for the $T_{\text {solvus }}$ agree fairly well with the experimental results at low Ru-concentrations (up to $\sim 6 \%$ ), while the discrepancy between the calculated and experimental results becomes significant for the high Ru-concentrations $(8 \sim 12 \%)$. We discuss that the discrepancy may be corrected by the CVM calculations with the large basic cluster including up to the 4th- $n n$ IE. [doi:10.2320/matertrans.M2017292]
\end{abstract}

(Received September 26, 2017; Accepted December 4, 2017; Published January 19, 2018)

Keywords: KKR-Green's function method, GGA, Cluster variation method, Fermi-Dirac distribution, thermal vibration, Debye-Grüneisen model, local lattice distortion, real-space cluster expansion

\section{Introduction}

Ab-initio calculations along with the cluster variation method (CVM) used to evaluate the configurational entropy have attracted broad attention as a theoretical approach for drawing the phase diagrams of alloys. ${ }^{1,2)}$ The phase stability and phase equilibria for many binary alloys have been successfully described by the CVM. For example, using the cluster variation method within the tetrahedron approximation (CVMT), the experimentally determined transition temperature $(1023 \mathrm{~K})$ of FePd alloys was reproduced very well $(1030 \mathrm{~K})$ by including the lattice thermal vibration effect. ${ }^{1)}$ The free energy in these calculations was divided into two parts (internal energy and configurational entropy). The effective cluster interaction energies (ECIEs), used in the calculations of the internal energy, were determined from the combination of the total energies of a set of selected ordered and elemental metals, following the Connolly-Williams approach. ${ }^{3)}$ For the configurational entropy by the CVMT, three fcc-based ordered alloys $\left(\mathrm{A}_{3} \mathrm{~B}, \mathrm{AB}, \mathrm{AB}_{3}\right)$ and two fcc elemental metals ( $\mathrm{A}$ and $\mathrm{B}$ ) were chosen for the $\mathrm{AB}$ alloys to determine the five kinds of ECIEs up to the tetrahedron formed from the 1st-nearest neighbor $(n n)$ pairs.

On the other hand, we also succeeded in reproducing the observed solvus temperatures, $T_{\text {solvus }}(820 \sim 860 \mathrm{~K})$, of the $\mathrm{Pd}_{1-c} \mathrm{Rh}_{c}$ fcc alloys $(0.09 \leq c \leq 0.12)$ by the free energy calculations based on the $\mathrm{CVMT}^{4)}$, although the local lattice distortion effect, existing even at $T=0 \mathrm{~K}$, and the thermal vibration effect were not taken into account. The Rh atoms were treated as impurities in Pd. It has been experimentally

*Corresponding author, E-mail: asato@sci.niihama-nct.ac.jp reported that the $\mathrm{Rh}$ impurities are segregated at low temperatures and disordered at high temperatures due to the configurational entropy. ${ }^{4,5)}$ As discussed later, the 2 4 body interaction energies (IEs) ${ }^{4)}$ among the Rh impurities, up to the tetrahedron formed by the 1st-nearest neighbor ( $n n$ ) pairs, were uniquely determined by the real-space cluster expansion (RSCE) based on the Korringa-Kohn-Rostoker Green's function (KKR) method for perfect and impurity systems, different from the Connolly-Williams approach. It is noted that the spherical potential approximation for the KKR method was used in the previous study. We found that the 2-body interaction between the $\mathrm{Rh}$ impurities in $\mathrm{Pd}$ is considerably short-range (see Fig. 4 in Ref. 4)), and the 3-body and 4-body interactions of the tetrahedron formed by the 1st- $n n$ pairs are considerably weak, as discussed in Ref. 4). We have shown that the observed $T_{\text {solvus }}$ of the $\mathrm{Pd}_{1-c} \mathrm{Rh}_{c}$ alloys may be well reproduced by the free energy calculations based on the CVMT, using these $2 \sim 4$ body IEs for the internal energy, if the attractive 1st-nn 2-body IE is renormalized by including the weakly repulsive $2 \sim 8$ th- $n n$ 2-body IEs. It is noted that the small reduction of the attractive 1st-nn 2-body IE is very important to accurately reproduce the observed $T_{\text {solvus }}$ of the $\mathrm{Pd}_{1-c} \mathrm{Rh}_{c}$ alloys, as shown in Ref. 4). These successful results based on the CVMT may be mainly due to the short-range nature of the IEs used in the calculations of the internal energies of the considered alloys.

In this paper, we present the $a b$-initio calculations for the observed $T_{\text {solvus }}(1000 \sim 1600 \mathrm{~K})$ of the $\operatorname{Pd}_{1-c} \operatorname{Ru}_{c}(0.03 \leq c \leq$ $0.12)$ fcc alloys ${ }^{5)}$, in which the Ru atoms are treated as impurities in Pd. In the present calculations, we carried out the ab-initio calculations based on the full-potential Korringa- 
Kohn-Rostoker Green's function (FPKKR) method, different from the previous calculations with the spherical potential approximation. Since the present approach is an approach from a dilute limit, we concentrated on studying the low-concentrated $\mathrm{Pd}_{1-c} \mathrm{Ru}_{c}$ alloys $(c \leq 0.12)$. We found that the 2-body interaction of the Ru impurities is very long-rang and considerably repulsive at the 2nd-, and 4th-, and 9th-nns in comparison to the strong attraction at the $1 \mathrm{st}-n n$, as decribed in this paper. ${ }^{6}$ ) Since the 2-body interaction between impurities in metals seem to be usually long-range, as shown in Refs. 4), 7), and 8), it is very important to study the $T_{\text {solvus }}$ of the $\mathrm{Pd}_{1-c} \mathrm{Ru}_{c}$ alloys as a typical example of binary alloys with the long-range 2-body interaction. It is also noted that the observed $T_{\text {solvus }}(1000 \sim 1600 \mathrm{~K})$ of the $\operatorname{Pd}_{1-c} \operatorname{Ru}_{c}(0.03 \leq$ $c \leq 0.12$ ) alloys significantly changes, different from the observed $T_{\text {solvus }}(820 \sim 860 \mathrm{~K})$ of the $\mathrm{Pd}_{1-c} \mathrm{Rh}_{c}(0.09 \leq c \leq 0.12)$ alloys. In the previous calculations for the Rh impurities in $\mathrm{Pd},{ }^{4)}$ the temperature $\left(T_{\mathrm{FD}}\right)$ of the Fermi-Dirac $(\mathrm{FD})$ distribution was fixed at $800 \mathrm{~K}$, which agrees with the observed $T_{\text {solvus }}(820 \sim 860 \mathrm{~K})$. We also found that the local lattice distortion effect may be more important for the Ru impurities in the Pd metal, compared to the $\mathrm{Rh}$ impurities in $\mathrm{Pd} .{ }^{6)}$ Thus, in addition to the 2-body long-range IEs up to the 20th- $n n$, in the present study we inevestigated the following three effects on the IEs of the Ru impurities in Pd; (1) the local lattice distortion for the 1st- $n n \mathrm{Ru}-\mathrm{Ru}$ IE, existing even at $T=$ $0 \mathrm{~K}$, (2) the lattice thermal vibration at finite temperatures, and (3) the $T_{\mathrm{FD}}$-dependence for the electron excitation due to the FD distribution.

In order to determine the $n$-body $(n=1 \sim 4)$ IEs among the $\mathrm{Ru}$ impurities in $\mathrm{Pd}$, we used the RSCE for the total energies of the alloys, ${ }^{9-12)}$ different from the Connolly-Williams Approach. ${ }^{3)}$ The present approach, while restricted to the dilute limit, considers only atomic configurations, the energy differences of which uniquely define the $n$-body IEs. The $n$-body IEs are successively determined from the low-order to high-order and are independent of the concentration. ${ }^{4,10)}$ On the other hand, the Connolly-Williams approach determines the ECIEs from different atomic configurations, in which the results of the supercell calculations with different configurations in the unit cell are fitted by models containing the ECIEs. Thus, the ECIEs depend on the selected set of ordered and elemental metals, as shown in Ref. 13). To verify the accuracy and convergence of the present approach for the Pd-rich PdRu alloys, it is very important to clarify the distance dependence of the 2-body (pair) IEs and the cluster-size dependence of the 3- and 4-body (trimer and tetramer) IEs among the $\mathrm{Ru}$ impurities in $\mathrm{Pd}$. We already examined how the total energies of the Pd-rich PdRu alloys can be accurately reproduced by the present RSCE including up to the 4-body IEs among the $\mathrm{Ru}$ impurities in $\mathrm{Pd}$ and found that the band calculation results for the $\mathrm{Pd}_{3} \mathrm{Ru}$ alloy in the $\mathrm{L}_{2}$ structure, as one example of the Pd-rich PdRu alloys, may be reproduced within the error of $1 \mathrm{mRy}$ per atom by the RSCE including the 2-body IEs up to the appropriate neighbors (for example, up to 20th- $n n$ ) and the 3-body IEs up to the appropriate size of the clusters (for example, up to the 3-body clusters in the 2 cubes in the fcc structure). The contribution from the $n$-body IEs becomes less and less with the increase in $n$, as discussed in Ref. 9). The calculated re- sults will be published in a subsequent paper. ${ }^{14)}$

In Sect. 2, we discuss the calculation procedures for the FPKKR method with the electron excitation effect due to the FD distribution and the thermal vibrational free energy. In Sect. 3 , the calculated results for the $T_{\text {solvus }}$ of the $\operatorname{Pd}_{1-c} \mathrm{Ru}_{c}$ $(0.03 \leq c \leq 0.12)$ alloys are discussed. We show that the calculated CVMT results agree fairly well with the observed $T_{\text {solvus }}$ for the low Ru-concentrations (up to $\sim 6 \%$ ), while the discrepancy (overestimation) between the calculated and experimental results becomes high for the high $\mathrm{Ru}-$ concentrations $(8 \sim 12 \%)$. We also show that this discrepancy may be corrected by the calculations based on the cluster variation method within the tetrahedron-octahedron approximation (CVMTO), in which the 2nd-nn IE is renormalized by including the 4th- $n n$ IE. We also found that, in addition to the local lattice distortion and the lattice thermal vibration effects, the $T_{\mathrm{FD}}$ dependence of the IEs due to the electron excitation of the FD distribution is very important to reproduce the observed $T_{\text {solvus }}(1000 \sim 1600 \mathrm{~K})$ of $\mathrm{Pd}_{1-c} \mathrm{Ru}_{c}(0.03 \leq c \leq$ $0.12)$. We will show that the electron excitation effect due to the FD distribution attractively works for the 2-body IEs and increases $T_{\text {solvus. }}$ Section 4 summarizes the main results of the present paper. It is also discussed that the CVM calculations for the larger basic clusters is strongly claimed to quantitatively study the phase diagrams of the long-range interaction systems, such as the present Pd-rich PdRu alloys.

\section{Calculation Method}

\subsection{Total energies of impurity systems}

The calculations for the total energy of the Ru impurities in Pd are based on the density functional theory (DFT) in the generalized gradient approximation (GGA). ${ }^{15,16)}$ In order to solve the Kohn-Sham equations, we used a multiple scattering theory in the form of the KKR Green's function method for the full-potentials (FP). We used the screened version of the FPKKR band calculations for the Pd host, which significantly simplifies the numerical calculations by introducing the short-range structural Green's functions. ${ }^{17,18)}$ In order to simplify the total energy calculations in the GGA formalism, we use the electronic densities self-consistently obtained by the local spin density approximation in the DFT. The accuracy of the present GGA calculations was discussed in Refs. 15) and 16).

The advantage of Green's function method is due to the introduction of the host Green's function and the embedding of point defects in an otherwise ideal crystal is correctly described, differently from the usual supercell and cluster calculations. It is noted that although the potential perturbation due to the defects is localized in the vicinity of the defects, the change in the wave functions due to the defects is delocalized over the whole space. The practical advantage in using Green's function method is to exploit this short-range nature of the defect potential. For example, in order to obtain the accurate and converged total energy of the impurity system without the lattice distortion in the fcc structure, it is sufficient to self-consistently redetermine only the potentials of the impurities and their 1st- $n n$ host atoms, if the total energy change due to the perturbed wave functions over the infinite space is correctly evaluated by using Lloyd's for- 
mula. ${ }^{16,19)}$ This is also very different from the supercell and cluster calculations in which all the potentials must be recalculated in each iteration cycle.

As discussed in Sect.3 and Appendix 2, in order to calculate the Ru-concentration dependence of the $T_{\text {solvus }}$ of the $\mathrm{Pd}_{1-c} \mathrm{Ru}_{c}$ alloys, we must first calculate the total energy difference between the disordered and segregated states. The total energy (per tetrahedron basic cluster of the 1st- $n n$ pairs) of the disordered state, within the CVMT approximation, is written as follows:

$$
E_{\mathrm{dis}}=2 \sum_{i, j, k, l} E_{i j k l} \omega_{i j k l}
$$

where $i, j, k$, and $l$ distinguish $\mathrm{Pd}$ or $\mathrm{Ru}, E_{i j k l}$ is the total energy (per tetrahedron basic cluster), and $\omega_{i j k l}$ is the cluster probability. $E_{i j k l}$ is calculated using the IEs up to the 4-body of the tetrahedron cluster, obtained in the RSCE. The definitions and calculation procedure of the IEs up to the 4-body are described in Refs. 4), 10), 12), and 14). The total energy of the segregated state is also expressed by using the cluster probability of the segregated state $\left(\omega_{\text {RuRuRuRu }}+\omega_{\text {PdPdPdPd }}=\right.$ $1, \mathrm{Ru}$-concentration $=\omega_{\mathrm{RuRuRuRu}}, \mathrm{Pd}$-concentration $=$ $\left.\omega_{\text {PdPdPdPd }}\right)$, as discussed in Appendix 2.

In the present study, we describe the $T_{\mathrm{FD}}$ dependence of the IEs due to the electron excitation of the FD distribution. As shown in Ref. 20), the introduction of the FD distribution into the ab-initio Green's function calculations is very useful to simplify the ground state calculations. Based on a contour integration in the complex energy plane, we evaluated the residues at a few Matsubara frequencies, while the remaining integral is analyzed by the Sommerfeld expansion. The imaginary part $(\delta)$ in the complex energy plane is related to $T_{\mathrm{FD}}$ of the FD distribution $\left(\delta=\pi k T_{\mathrm{FD}}\right)$. The total energy is calculated by the grand canonical potential for which a simple formula is given. Since the total energy change due to the electron excitation is usually expected to be negligible, the FD distribution, usually with $T_{\mathrm{FD}}=800 \mathrm{~K}$ for metals, has been expediently used to efficiently perform the ground state calculations. ${ }^{15,16,20)}$ In the present study, we evaluated the $T_{\mathrm{FD}}$-dependence for the IEs among the Ru impurities in $\mathrm{Pd}$ and show that the effect due to the FD distribution becomes very important to reproduce the concentration dependence of the observed high $T_{\text {solvus. }}$.

In the present study, we also evaluated the local lattice distortion effect for the 1 st- $n n$ Ru-Ru IE in Pd. ${ }^{21,22)}$ The local lattice distortion energy is defined as the total energy change caused by the lattice distortion around the impurities. ${ }^{23}$ It is noted that the local lattice distortion energy for the $1 \mathrm{st}-n n \mathrm{Ru}-\mathrm{Ru}$ IE is defined as the difference between the local lattice distortion energies of the two states: (1) the final state where two impurities are located at the 1st- $n n$ sites, and (2) the initial one where both impurities are infinitely apart. As discussed in Refs. 21)-24), the treatment of the lattice distortion is very cumbersome in the present KKR method. In order to study the lattice distortion effect, we must self-consistently redetermine the potentials in the larger region compared to the calculations for the impurity systems without the local lattice distortion, because the potentials of the host atoms surrounding the displaced host atoms may be strongly perturbed. Since the local lattice distortion up to the 1st- $n n$ host atoms around the 1st-nn Ru-Ru impurity pair in $\mathrm{Pd}$ is taken into account in the present calculations, we self-consistently redetermined the potentials in the 102-atom impurity cluster in Pd, as discussed in Ref. 24). In the present calculations, the positions of the displaced atoms are determined by the condition of the Hellmann-Feynman forces $=0$. The detailed discussion for the local lattice distortion and the accuracy of the present calculations are reported in Ref. 21).

\subsection{Vibrational free energy}

In addition to the local lattice distortion effect due to the difference in the atomic size, existing even at $T=0 \mathrm{~K}$, the effect of the lattice softening due to the thermal vibration at finite temperatures is considered in the present study. The vibrational free energy for the lattice softening is calculated by using the Debye-Grüneisen model which is based on the quasi-harmonic approximation. By following Ref. 1), the vibrational free energy, $F_{\text {vib }}^{(n)}(a, T)$, of each phase $(n)$ consisting of the basic cluster, $\mathrm{Pd}_{4-} \mathrm{Ru}_{n}(n=0 \sim 4)$, used in the CVMT (see Fig. 2 in Ref. 4), $\mathrm{H}=\mathrm{Pd}$ and $\mathrm{I}=\mathrm{Ru}$ ), is written as follows:

$$
F_{\mathrm{vib}}^{(n)}(a, T)=E_{\mathrm{vib}}^{(n)}(a, T)-T S_{\mathrm{vib}}^{(n)}(a, T)
$$

where $E_{\mathrm{vib}}^{(n)}(a, T)$ is the vibrational internal energy and $S_{\mathrm{vib}}^{(n)}(a, T)$ is the vibrational entropy depending on the lattice parameter $(a)$, temperature $(T)$, and the phase $(n)$ specified by the basic cluster $\left(\mathrm{Pd}_{4-n} \mathrm{Ru}_{n}\right)$. These terms are given by the following formulae:

$$
\begin{array}{r}
E_{\mathrm{vib}}^{(n)}(a, T)=\frac{9}{8} k_{B} \cdot \Theta_{D}^{(n)}+3 k_{B} \cdot T \cdot D\left(\frac{\Theta_{D}^{(n)}}{T}\right) \\
S_{\mathrm{vib}}^{(n)}(a, T)=3 k_{B} \cdot\left[\frac{4}{3} D\left(\frac{\Theta_{D}^{(n)}}{T}\right)-\ln \left\{1-\exp \left(-\frac{\Theta_{D}^{(n)}}{T}\right)\right\}\right]
\end{array}
$$

where $\Theta_{D}^{(n)}$ is the Debye temperature of the phase $(n)$ consisting of the basic cluster $\mathrm{Pd}_{4-n} \mathrm{Ru}_{n}$ (fcc structure for $\mathrm{Pd}_{4}$ and $\mathrm{Ru}_{4}, \mathrm{~L} 1_{2}$ for $\mathrm{Pd}_{3} \mathrm{Ru}$ and $\mathrm{PdRu_{3 }}$, and $\mathrm{L} 1_{0}$ for $\mathrm{Pd}_{2} \mathrm{Ru}_{2}$ ), determined by using the lattice parameter dependence of the total energy. The calculation procedure for the total energy $\left(E_{\mathrm{Pd}_{4-n}} \mathrm{Ru}_{n}\right)$ per basic cluster is described in Appendix 1. $D(x)$ is a Debye function and the first term of the vibrational internal energy (eq. (3)) represents the zero-point energy.

\section{Solvus Temperatures of $\operatorname{Pd}_{1-c} \mathbf{R} \mathbf{u}_{c}$}

We discuss the ab-initio calculations for the $\mathrm{Ru}$ concentration dependence of the $T_{\text {solvus }}$ of the $\mathrm{Pd}_{1-c} \mathrm{Ru}_{c}$ $(0.03 \leq c \leq 0.12)$ alloys. In the present study, we calculated the free energy difference (eq. (5)) between the disordered and segregated states,

$$
\begin{gathered}
\Delta F(a, T)=\Delta E(a, T)-T S_{\mathrm{conf}}(a, T) \\
\Delta E(a, T)=E_{\mathrm{dis}}(a, T)-E_{\mathrm{seg}}(a, T)
\end{gathered}
$$

where $E_{\mathrm{dis}}(a, T)$ and $E_{\mathrm{seg}}(a, T)$ are the internal energies corresponding to the disordered and segregated states, respectively, depending on the lattice parameter $(a)$ and tempera- 
ture $(T)$, and include the contributions from the electron excitation due to the FD distribution and the lattice thermal vibration effect (eq. (2)) based on the Debye-Grüneisen model, discussed in Sect. 2. The $S_{\text {conf }}(a, T)$ is the configurational entropy calculated by the CVMT. The $S_{\text {conf }}(a, T)$ and $\Delta E(a, T)$ are expressed by the cluster probabilities $\left\{\omega_{i j k l}\right\}$ of the tetrahedron, where $i, j, k$, and $l$ distinguish $\mathrm{Pd}$ or $\mathrm{Ru}$. Thus, by minimizing the free energy difference (eq. (5)) with respect to the cluster probabilities $\left\{\omega_{i j k l}\right\}$, we can determine the cluster probabilities in the equilibrium state. The calculation procedure for the minimization of $\Delta F(a, T)$ is described in Appendix 2. The Ru-concentration limit to a given temperature $\left(T_{\text {solvus }}\right)$ is calculated by using the calculated values for $\left\{\omega_{i j k l}\right\}$, as the Ru-concentration $=\sum_{j, k, l=\mathrm{Pd}, \mathrm{Ru}} \omega_{\mathrm{Ru}, j k l}$. In the present calculations, the equilibrium lattice parameter of $\mathrm{Pd}_{1-c} \mathrm{Ru}_{c}$ at a given $T$ is approximated by the equilibrium lattice parameter of the Pd-host determined by the minimization of $E_{\mathrm{Pd}_{4}}(a, T)$ with the electron excitation due to the FD distribution and the lattice thermal vibration effect, since the difference between the equilibrium lattice parameters $(a(T, c)$ and $a(T, 0))$ of $\mathrm{Pd}_{1-c} \mathrm{Ru}_{c}$ and $\mathrm{Pd}$ at a given $T$ may be small for the low Ru-concentration, as seen in Fig. 3. The error caused by this approximation will be discussed in the last paragraph of this section.

In the CVMT approximation, we can treat IEs exactly up to the 4-body in a tetrahedron formed from the 1st- $n n$ pairs. However, we have found that the 2-body interaction is longrange for the $\mathrm{Ru}$ impurities in $\mathrm{Pd}$, as shown in Fig. 1, and may be important up to the 10th- $n n$ interaction; the positive (repulsive) IEs at the 2nd-, 4th-, and 9th-nns are comparatively large. ${ }^{6)}$ It is noted that the $1 \mathrm{st}(0.5,0.5,0)-$, 4 th $(1,1,0)$-, and 9th-a(1.5,1.5,0)- nns align along the $<110>$ direction, although we have not understood the micro-mechanism of the rather large repulsion at the 9th-a $n n$. Thus, as the first approximation, we renormalized the strong and attractive 1st- $n n$ IE (negative value) as follows, as discussed in Ref. 4):

$$
\begin{aligned}
\Delta \tilde{E}_{1}^{2-\text { body }}= & \Delta E_{1}^{2-\text { body }}+\frac{6}{12} \Delta E_{2}^{2-\text { body }}+\frac{24}{12} \Delta E_{3}^{2-\text { body }}+\cdots \\
& +\frac{24}{12} \Delta E_{10}^{2-\text { body }}
\end{aligned}
$$

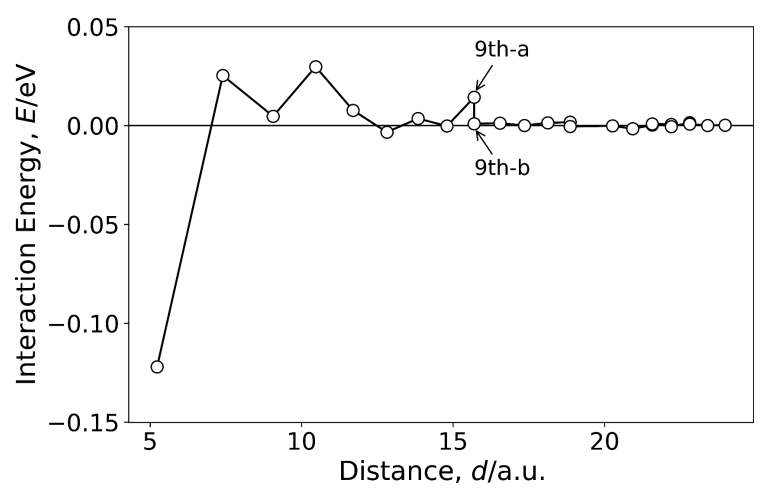

Fig. 1 Calculated results for the distance dependence (1 20th- $n n)$ of the $\mathrm{Ru}-\mathrm{Ru}$ IEs in Pd for the lattice parameter of 7.40 a.u., with the FD distribution at $T_{\mathrm{FD}}=800 \mathrm{~K}$. There are two nonequivalent sites for the 9th-, 13 th-, 16th-, 17th-, and 18th-nns. The 9th-a and 9th-b $n n$ sites are (1.5, $1.5,0)$ and $(2,0.5,0.5)$ in the unit of the lattice parameter $(7.40$ a.u.), respectively. See the text for details. where the coefficient in the $n$ th- $n n$ term is the ratio of the coordination number of the $n$ th- $n n$ shell to that of the 1 st- $n n$ shell. It is noted that the sum total of the long-range 2-body IEs up to the 10th- $n n$ is maintained in the renormalized 1st-nn IE. In the present paper, the CVMT calculations with the 1st-nn IE renormalized by the IEs up to the 10th- $n n$ are written as CVMT10. The lattice thermal vibration effect for the renormalized 1st- $n n$ IE is taken into account in the present calculations, as discussed in the last paragraph of Appendix 1.

The 3-body and 4-body IEs are listed in Table 1 as their lattice parameter $(a)$ dependence and $T_{\mathrm{FD}}$-dependence for the FD distribution. These values are considerably small compared to the 2-body IEs. It is also noted that the contributions from the 3-body and 4-body are proportional to the 3rd- and 4th-power of the Ru-concentration. Thus, in the present study for the low Ru-concentration, we do not consider the contributions from the 3-body and 4-body IEs of the larger clusters. Figure 2 shows the calculated results for the distance $(1 \sim 10$ th- $n n)$ and $T_{\mathrm{FD}}(=800,1000,1200,1400$, $1600 \mathrm{~K}$ )-dependence of the 2-body IEs, for the lattice parameter of 7.40 a.u. We find that the $T_{\mathrm{FD}}$-dependence is not

Table 1 Calculated results (in eV) for the 3-body and 4-body IEs among the Ru impurities in Pd, shown in Fig. 2 ( $\mathrm{I}=\mathrm{Ru}, \mathrm{H}=\mathrm{Pd}$ ) in Ref. 4), for seven lattice parameters ( $a=6.8 \sim 8.0$ a.u., in interval of 0.2 a.u.) and at three temperatures $\left(T_{\mathrm{FD}}=800,1200,1600 \mathrm{~K}\right)$ for the FD distribution. See the text for details.

\begin{tabular}{ccccccc}
\hline \multirow{2}{*}{$a$} & \multicolumn{2}{c}{$T_{\mathrm{FD}}=800 \mathrm{~K}$} & \multicolumn{2}{c}{$1200 \mathrm{~K}$} & \multicolumn{2}{c}{$1600 \mathrm{~K}$} \\
\cline { 2 - 7 } (a.u.) & $E_{\text {int }}^{3 \text {-body }}$ & $E_{\text {int }}^{4-\text { body }}$ & $E_{\text {int }}^{3-\text { body }}$ & $E_{\text {int }}^{4-\text { body }}$ & $E_{\text {int }}^{3-\text { body }}$ & $E_{\text {int }}^{4-\text { body }}(\mathrm{eV})$ \\
\hline 6.8 & 0.0128 & 0.0085 & 0.0140 & 0.0092 & 0.0151 & 0.0090 \\
7.0 & 0.0125 & 0.0085 & 0.0136 & 0.0090 & 0.0146 & 0.0084 \\
7.2 & 0.0124 & 0.0086 & 0.0134 & 0.0086 & 0.0142 & 0.0076 \\
7.4 & 0.0125 & 0.0085 & 0.0134 & 0.0080 & 0.0138 & 0.0067 \\
7.6 & 0.0128 & 0.0084 & 0.0133 & 0.0073 & 0.0132 & 0.0057 \\
7.8 & 0.0131 & 0.0081 & 0.0132 & 0.0064 & 0.0125 & 0.0049 \\
8.0 & 0.0134 & 0.0076 & 0.0129 & 0.0056 & 0.0117 & 0.0041 \\
\hline
\end{tabular}

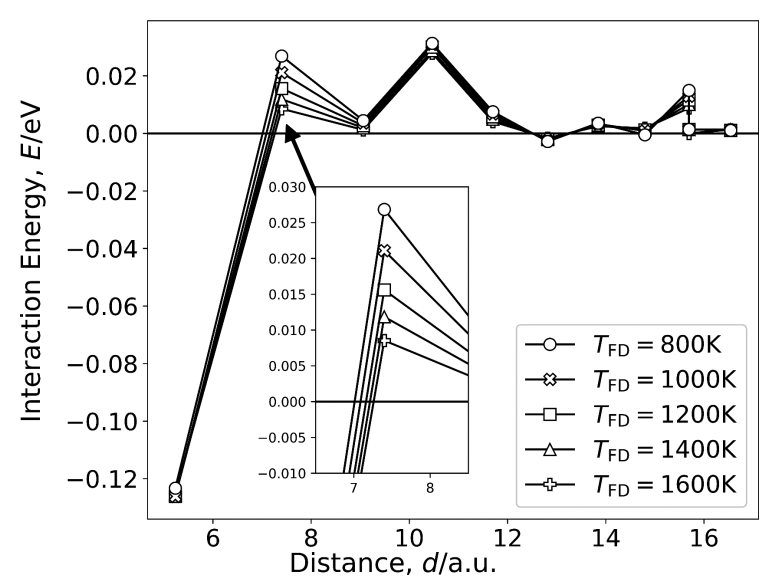

Fig. 2 Calculated results for the distance (1 10th- $n n)$ dependence of the $\mathrm{Ru}-\mathrm{Ru}$ IEs in Pd with the FD distribution at $T_{\mathrm{FD}}=800,1000,1200$, 1400 , and $1600 \mathrm{~K}$. The lattice parameter is fixed at 7.40a.u. The repulsive 2nd- $n n$ IE becomes smaller and smaller with the increasing $T_{\mathrm{FD}}$. The enlarged view around the 2nd- $n n$ is inserted in Fig. 2. See the text for details. 
as small as we have usually been expecting it to be. ${ }^{25)}$ It is noted that the repulsive interaction at the 2nd- $n n$ becomes considerably weak with the increasing $T_{\mathrm{FD}}$. This means that the temperature effect of the electron excitation due to the FD distribution attractively works for the 2-body IEs of the $\mathrm{Ru}$ impurities in Pd. As shown later, this effect is very important to reproduce the observed $T_{\text {solvus }}$.

The vibrational internal energy (eq. (3)) and vibrational entropy (eq. (4)) for the basic cluster $\left(\mathrm{Pd}_{4-n} \mathrm{Ru}_{n}, n=0 \sim 4\right)$ used in the CVMT were calculated using the Debye temperatures which were determined from the lattice parameter dependence of the total energies per $\mathrm{Pd}_{4-n} \mathrm{Ru}_{n}$, as discussed in Sect. 2.2. The lattice parameter dependences $(6.8 \sim 8.0$ a.u.) of the total energies $\left(E_{\mathrm{Pd}_{4-n}} R u_{n}, n=0 \sim 4\right)$ at $T_{\mathrm{FD}}=$ $1200 \mathrm{~K}$ without the thermal vibration effect are shown in Fig. 3(a), and those with the thermal vibration effect at $T_{\text {vib }}=$ 800, 1200 and $1600 \mathrm{~K}$ are shown in Figs. 3(b), (c) and (d). For the lattice parameter dependence, the FPKKR calculations were done in intervals of 0.1 a.u. Table 2 shows the calculated results for the renormalized 1st- $n n$ IE with the thermal vibration effect. It is noted that the negative values of IE become larger with the increasing $T_{\text {vib }}$. This means that the thermal vibration effect works attractively.

In the present calculations, we also take into account the local lattice distortion effect for the 1 st- $n n$ Ru-Ru IE. The calculated lattice distortion energies (negative values) for the 1 st- $n n$ Ru-Ru IEs at $a=7.5$ and 7.6 a.u., with the FD distribution at $T_{\mathrm{FD}}=800,1000,1200,1400$ and $1600 \mathrm{~K}$, are listed in Table 3. We found that the local lattice relaxation effect also works attractively for the 1st- $n n \mathrm{Ru}-\mathrm{Ru}$ interaction and that both the dependences on $a$ and on $T_{\mathrm{FD}}$ are very small. In the following calculations for $T_{\text {solvus }}$, the lattice distortion energies for the equilibrium lattice parameters $\left(a_{o}=7.54\right.$,
$7.56,7.59,7.61,7.63$ a.u.), corresponding to the given temperatures $(T=800,1000,1200,1400,1600 \mathrm{~K})$, are estimated by interpolating or extrapolating the two values at 7.5 and 7.6 a.u., as listed in Table 3.

We now discuss the calculated results for the $\mathrm{Ru}-$ concentration dependence of the $T_{\text {solvus }}$ of the $\mathrm{Pd}_{1-c} \mathrm{Ru}_{c}$ $(0.03 \leq c \leq 0.12)$ alloys. In order to clarify the lattice thermal vibration effect, we carried out two kinds of calculations without and with the thermal vibration effect. For the calculations without the thermal vibration effect, the lattice parameter is constant at 7.46 a.u., obtained by the FPKKR cal-

Table 2 Calculated results (in eV) for the renormalized 1st- $n n$ IEs with the thermal vibration at $T_{\mathrm{vib}}=800,1200$, and $1600 \mathrm{~K}$. These calculations were executed with the FD distribution at $T_{\mathrm{FD}}=800,1200$, and $1600 \mathrm{~K}$, respectively. See the text for details.

\begin{tabular}{cccc}
\hline \multirow{2}{*}{$T_{\mathrm{FD}}$} & \multicolumn{3}{c}{ renormalized 1st-nn IEs $(\mathrm{eV})$} \\
\cline { 2 - 4 } & $T_{\text {vib. }}=800 \mathrm{~K}$ & $1200 \mathrm{~K}$ & $1600 \mathrm{~K}$ \\
\hline $800 \mathrm{~K}$ & -0.0662 & -0.0681 & -0.0705 \\
\hline $1200 \mathrm{~K}$ & -0.0825 & -0.0833 & -0.0841 \\
\hline $1600 \mathrm{~K}$ & -0.0987 & -0.0993 & -0.1000 \\
\hline
\end{tabular}

Table 3 Calculated results (in eV) for the local lattice distortion energies for the 1st-nn Ru-Ru IEs in Pd. These calculations were executed for two lattice parameters ( $a=7.5,7.6$ a.u.) with the FD distribution at $T_{\mathrm{FD}}=800,1000,1200,1400$ and $1600 \mathrm{~K}$, respectively. See the text for details.

\begin{tabular}{cccccc}
\hline$a$ & \multicolumn{5}{c}{ local lattice relaxation energies (eV) } \\
\cline { 2 - 6 } (a.u.) & $T_{\mathrm{FD}}=800 \mathrm{~K}$ & $1000 \mathrm{~K}$ & $1200 \mathrm{~K}$ & $1400 \mathrm{~K}$ & $1600 \mathrm{~K}$ \\
\hline 7.5 & -0.0336 & -0.0340 & -0.0342 & -0.0344 & -0.0346 \\
\hline 7.6 & -0.0346 & -0.0349 & -0.0353 & -0.0367 & -0.0361 \\
\hline
\end{tabular}

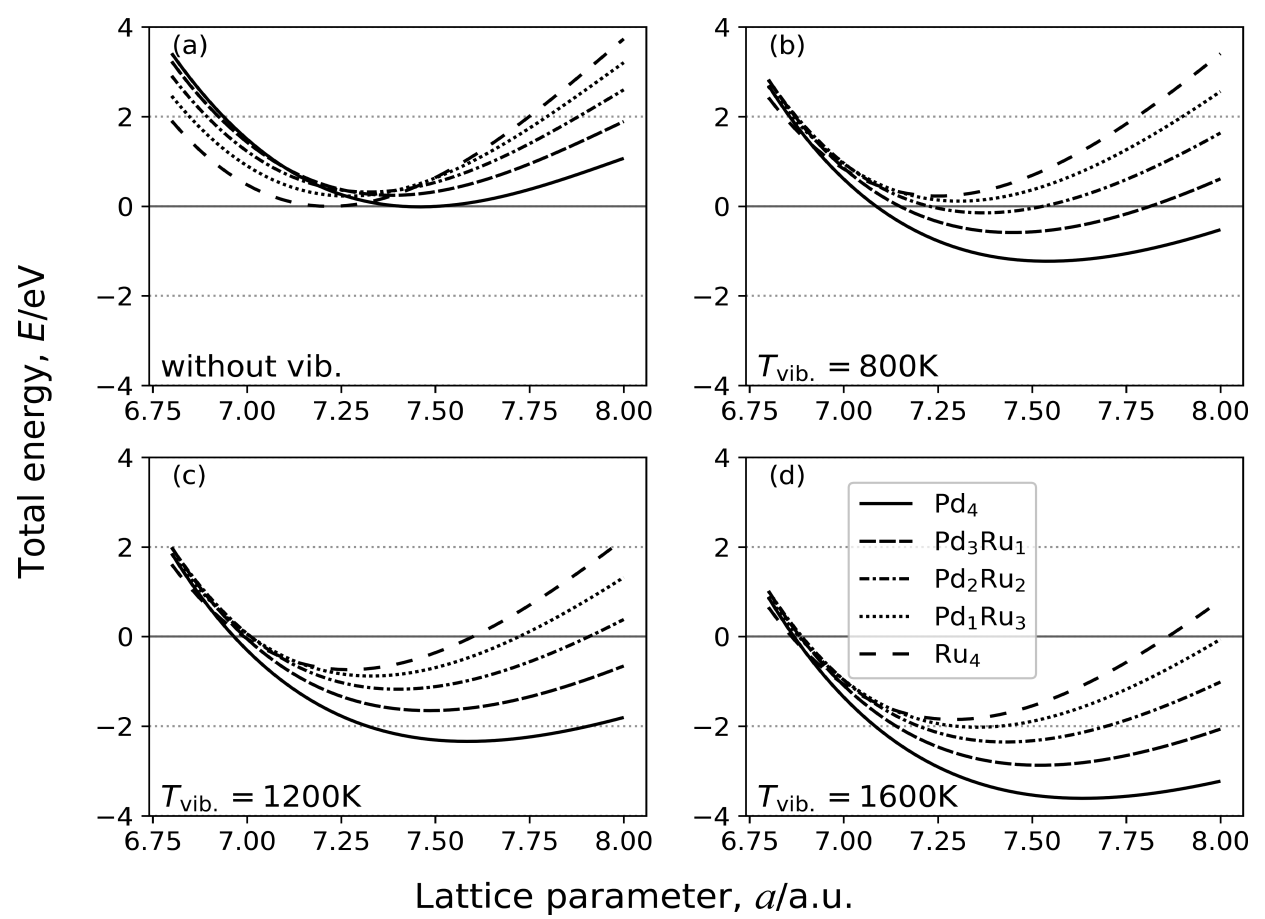

Fig. 3 Lattice parameter dependence (6.8 8.0a.u.) of the total energies $\left(E_{\mathrm{Pd}_{4-n} \mathrm{Ru}_{n}}\right)$ per basic cluster of five phases, $\mathrm{Pd}_{4-n} \mathrm{Ru}_{n}(n=0 \sim 4)$, discussed in Appendix 1: (a) total energies without thermal vibration effect; (b), (c) and (d) those with the thermal vibration effect at $T_{\text {vib. }}=800$, 1200 and $1600 \mathrm{~K}$, respectively. All the calculations were executed with the FD distribution at $T_{\mathrm{FD}}=1200 \mathrm{~K}$. See the text for details. 

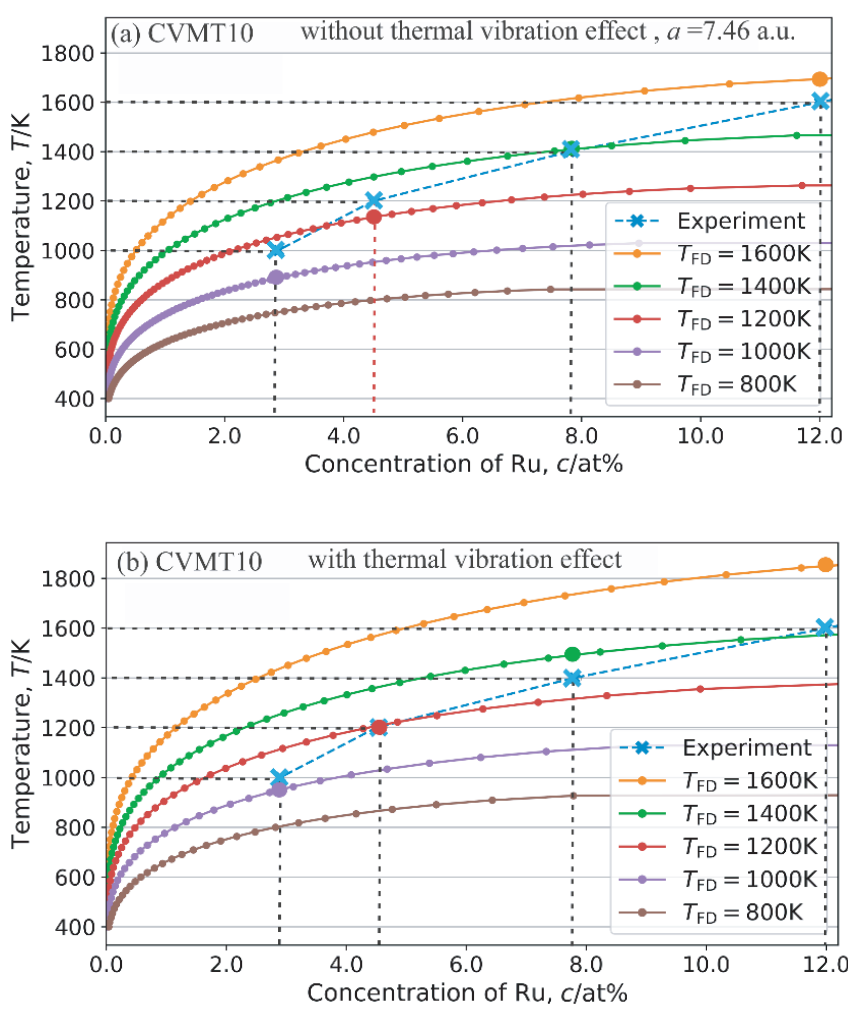

Fig. 4 Concentration dependence of the $T_{\text {solvus }}$ of the $\operatorname{Pd}_{1-c} \operatorname{Ru}_{c}(0.3 \leq c \leq$ 0.12) alloys obtained by the two kinds of CVMT10 calculations: (a) without and (b) with the thermal vibration effect. The calculated results are shown for the five temperatures $\left(T_{\mathrm{FD}}=800,1000,1200,1400\right.$, and $1600 \mathrm{~K})$. See the text for details.

Table 4 Experimental results for the $\mathrm{Ru}$-concentration dependence of the $T_{\text {solvus }}$ of the $\mathrm{Pd}_{1-c} \mathrm{Ru}_{c}$ alloys. ${ }^{5}$

\begin{tabular}{ccccc}
\hline$c \times 100(\%)$ & 2.7 & 4.4 & 7.8 & 12.0 \\
\hline$T_{\text {solvus }}(\mathrm{K})$ & 1000 & 1200 & 1400 & 1600 \\
\hline
\end{tabular}

culations at $T_{\mathrm{FD}}=800 \mathrm{~K}$. We found that the equilibrium lattice parameter is almost independent of $T_{\mathrm{FD}}$. Figure 4 (a) shows the CVMT10 results without the thermal vibration effect using the IEs at $T_{\mathrm{FD}}=800,1000,1200,1400$, and $1600 \mathrm{~K}$ together with the experimental results listed in Table $4 .{ }^{5)}$ It was found that $T_{\text {solvus }}$ become higher and higher with the increasing $T_{\mathrm{FD}}$. These results are easily understood by considering that the IEs (positive values, repulsion) at the 2nd-, 4th, 9th-a-nns become smaller (less repulsive) with the increasing $T_{\mathrm{FD}}$ by the effect of the electron excitation due to the FD distribution, as already discussed. It is noted that, in the present calculations, the observed $T_{\text {solvus }}(\times)$ for a given $\mathrm{Ru}$-concentration should agree with the calculated result (O) obtained by using $T_{\mathrm{FD}}=T_{\text {solvus }}$. We then compared the calculated results $(\mathbf{O})$ with the experimental results $(\mathbf{x})$ for $T_{\text {solvus }}$ to the given Ru-concentration, listed in Table 4. For the observed $T_{\text {solvus }}=1000$ and $1200 \mathrm{~K}$, corresponding to the Ru-concentration limits of $2.7 \%$ and $4.4 \%$, the CVMT10 calculations underestimate the experimental results by $\sim 110$ and $\sim 80 \mathrm{~K}$, respectively. For the observed $T_{\text {solvus }}=1400 \mathrm{~K}$, corresponding to the Ru-concentration limit of $7.8 \%$, the CVMT10 result agrees with the observed result. On the other hand, for the observed $T_{\text {solvus }}=1600 \mathrm{~K}$, corresponding to the Ru-concentration limit of $12.0 \%$, the CVMT10 calculations overestimate the experimental result by $\sim 100 \mathrm{~K}$.

Figure 4(b) shows the CVMT10 results with the thermal vibration effect. We found that the calculated $T_{\text {solvus }}$ considerably increases with the thermal vibration effect, resulting in the good agreement with the observed $T_{\text {solvus }}$ for the low Ru-concentration up to $\sim 4 \%$ and the higher overestimation $(100 \sim 260 \mathrm{~K})$ for the higher Ru-concentrations $(8 \sim 12 \%)$, corresponding to the higher $T_{\text {solvus }}=1400 \sim 1600 \mathrm{~K}$. The increase in $T_{\text {solvus }}$ is understood by considering that the renormalized attractive $1 \mathrm{st}-n n$ IE (negative value, attraction) increases with the increasing $T_{\mathrm{vib}}$, for examples, as -0.066 , -0.083 , and $-0.100 \mathrm{eV}$ for $T_{\mathrm{vib}}=T_{\mathrm{FD}}=800,1200$, and $1600 \mathrm{~K}$, respectively, as listed in Table 2 . As a result, we can conclude that, as shown in Fig. 4(b), the CVMT10 calculations reproduce fairly well (within the error of $\sim 50 \mathrm{~K}$ ) the observed $T_{\text {solvus }}$ for the low Ru-concentration up to $\sim 6 \%$, while the discrepancy (overestimation) between the calculated and observed results becomes greater for the higher $\mathrm{Ru}$-concentrations $(8 \sim 12 \%)$. The overestimation is as high as $\sim 260 \mathrm{~K}$ around the observed $T_{\text {solvus }}=1600 \mathrm{~K}$. This discrepancy may be caused by the CVMT10 calculations with the insufficient renormalization (eq. (7)) of the long-range 2-body IEs.

According to the Monte Carlo simulations of Schweika, ${ }^{26,27)}$ the overestimation due to the insufficient renormalization (eq. (7)) becomes higher and higher with the increasing concentration of the minor element, for the alloys with the competitive 2-body IEs (the strongly attractive IE at the 1st- $n n$ and the considerably repulsive IE at the 2nd- $n n$ ), being similar to the present $\mathrm{Pd}_{1-c} \mathrm{Ru}_{c}$ alloys. It now appears obvious that the suitable treatment for the repulsive IEs at the 2nd- and 4th-, and 9th-nns is important to correct the high discrepancy toward the higher $T_{\text {solvus, }}$ especially for the 4th- $n n$ IE because it is highly repulsive in the high $T_{\mathrm{FD}}$ $(1400 \sim 1600 \mathrm{~K})$ and the 4th-nns brings the higher coordination number (12) compared to the 2nd-nns.

In order to clarify the influence of the renormalization for the 2-body IEs on the overestimation of $T_{\text {solvus, }}$, we carried out the calculations based on the cluster variation method within the tetrahedron-octahedron approximation (CVMTO), in which the 1st- and 2nd-nn IEs can be taken into account. We treat the repulsive IE at the 2nd-nn, independently from the attractive IE at the 1st-nn, and renormalize the 1st- $n n$ IE by including the 3 10th- $n n$ IEs (eliminating the 2nd-nn part from eq. (7)). In the present paper, the CVMTO calculations with the 1 st- $n n$ IE renormalized by the IEs up to the 10th- $n n$ are written as CVMTO10. The thermal vibration effect for the 2nd-nn IE is calculated by the way similar to that discussed in Sect.2.2 with the basic cluster $\left(\mathrm{Pd}_{6-n} \mathrm{Ru}_{n}\right)$ of the octahedron. However, we found by comparing Fig. 5(a) with Fig. 4(b) by the CVMT10 calculation, that the improvement by introducing the CVMTO10 approximation is inadequate, especially for the high $T_{\text {solvus }}$ around $1600 \mathrm{~K}$, although the overestimation around $T_{\text {solvus }}=$ $1400 \sim 1600 \mathrm{~K}$ is reduced from $100 \sim 260 \mathrm{~K}$ to $85 \sim 225 \mathrm{~K}$. It is noted that the $T_{\text {solvus }}$ below $1200 \mathrm{~K}$, corresponding to the low $\mathrm{Ru}$-concentration $(2 \sim 4 \%)$, is almost unchanged. The inadequacy around $T_{\text {solvus }}=1600 \mathrm{~K}$ is mainly caused by the 
fact that the repulsive (positive) IE at the 2nd- $n n$ becomes very small with the increasing $T_{\mathrm{FD}}$, as shown in Fig. 2 . In order to obtain a good agreement with the experimental results for the high $T_{\text {solvus }}$ around $1600 \mathrm{~K}$, we may need the correct treatment not only for the 2nd-nn IE, but also for the strongly repulsive 4 th- $n n$ IE even at the high $T_{\mathrm{FD}}$.

In order to suitably include the effect of the repulsive 4 th- $n n$ IE, we then renormalized the repulsive 2 nd- $n n$ IE by including the 4th- $n n$ IE, while the 1 st- $n n$ IE by including the 3- and 5 10th- $n n$ IEs (eliminating the 2nd- and 4th- $n n$ parts from eq. (7)), since we cannot exactly take into account the 4th- $n n$ IE by the CVMTO calculation. The CVMTO10 results with this renormalization $(2 \mathrm{nd} \leftarrow 4$ th) for the 1 st and 2nd IEs are shown in Fig. 5(b). In contrast to the result in Fig. 4 (b) (the overestimation $(\sim 260 \mathrm{~K})$ around $T_{\text {solvus }}=$ $1600 \mathrm{~K})$, the results in Fig. 5(b) (2nd $\leftarrow 4$ th) agree very well with the experimental results. It is noted that the overestimation around $T_{\text {solvus }}=1600 \mathrm{~K}$ is less than $\sim 50 \mathrm{~K}$. Figure $5(\mathrm{~b})$ also shows the calculated results obtained by the different renormalization $(2 \mathrm{nd} \leftarrow 3 \sim 5$ th) for the 1 st- and 2 nd- $n n$ IEs, where the 2nd-nn IE is renormalized by including the $3 \sim 5$ th- $n n$ IEs and the 1st- $n n$ IE is renormalized by including the $6 \sim 10$ th- $n n$ IEs. The calculated result shows the low underestimation $(\sim 55 \mathrm{~K})$ around $T_{\text {solvus }}=1600 \mathrm{~K}$. This means that the CVMTO10 calculations with the suitably renormalized 1st- $n n$ and 2nd- $n n$ IEs may reproduce the observed $T_{\text {solvus }}$ up to the higher Ru-concentration $(\sim 12 \%)$. Thus, we may conclude that the exact treatment for the repulsive
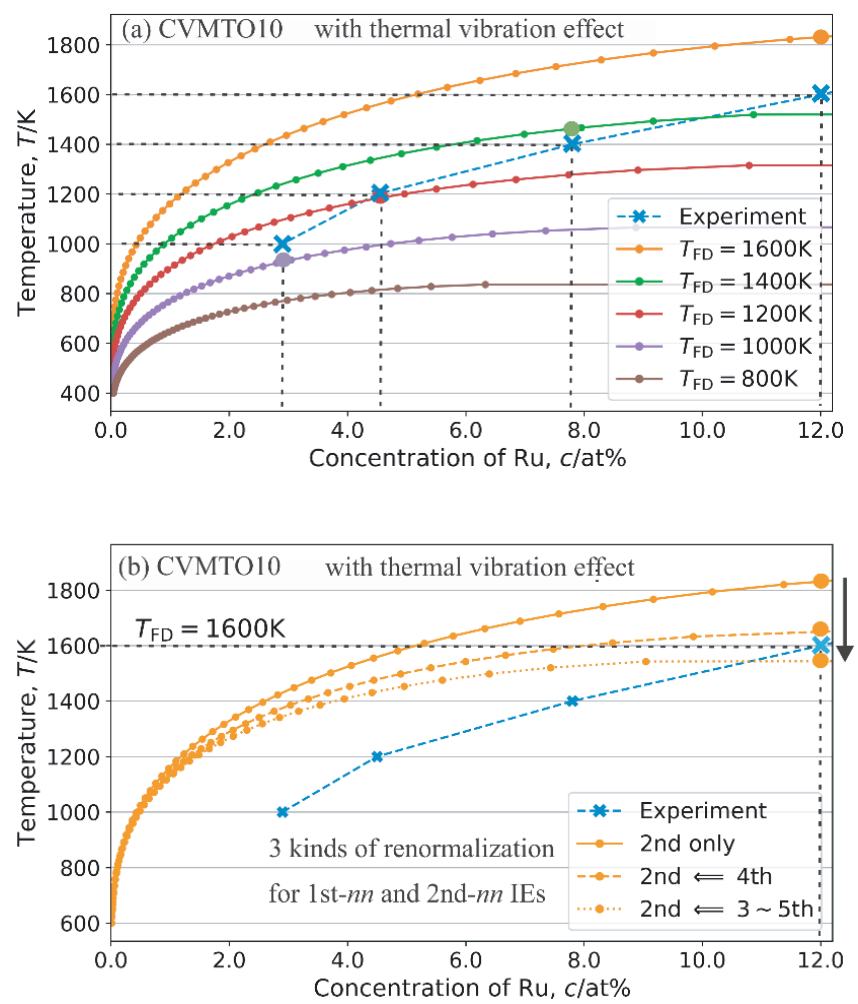

Fig. 5 Concentration dependence of the $T_{\text {solvus }}$ of the $\operatorname{Pd}_{1-c} \operatorname{Ru}_{c}(0.3 \leq c \leq$ 0.12) alloys obtained by the CVMTO10 calculation. In (a), the CVMTO10 results with the 1st-nn IE renormalized by the 3 10th- $n n$ IEs except the 2nd- $n n$ IE, and with the FD distribution at $T_{\mathrm{FD}}=800$, $1000,1200,1400$, and $1600 \mathrm{~K}$, are shown. In (b), the CVMTO10 results due to the different renormaization for the 1st- and 2nd- $n n$ IEs are shown. For (b), $T_{\mathrm{FD}}$ is fixed at $1600 \mathrm{~K}$. See the text for details. 4th-nn IE, beyond the CVMTO approximation, is very important to quantitatively study the observed $T_{\text {solvus }}$ around $1400 \sim 1600 \mathrm{~K}$, corresponding to the high Ru-concentration limits $(8 \sim 12 \%)$.

We now discuss the error caused by using the equilibrium lattice parameter $(a(T, 0))$ of $\mathrm{Pd}$ instead of that $(a(T, c))$ of $\mathrm{Pd}_{1-c} \mathrm{Ru}_{c}$ at a given $T$. In order to take into account the $\mathrm{Ru}-$ concentration dependence of the equilibrium lattice parameter of $\mathrm{Pd}_{1-c} \mathrm{Ru}_{c}$, we prepared the equilibrium lattice parameter $a(T, c)$ by fitting the five equilibrium lattice parameters of $\mathrm{Pd}_{4-n} \mathrm{Ru}_{n} \quad(n=0 \sim 4)$, shown in Fig. 3. The $\mathrm{Ru}-$ concentration limit at a given $T$ is self-consistently determined using the obtained $a(T, c)$. The CVMT10 results for the $T_{\text {solvus }}$ are shown in Fig. 6 and compared to the CVMT10 results in Fig. 4(b), being obtained using the lattice parameters $(a(T, 0))$ of Pd. For the low Ru-concentrations $(c=$ $0.027 \sim 0.044)$, the calculated results shown in Fig. 6 are almost the same to those obtained using the lattice parameter $a(T, 0)$ of Pd and agree very much with the experimental results. On the other hand, for the higher Ru-concentrations, we found that the discrepancies between the calculated and experimental results are reduced by $35 \mathrm{~K}(100 \mathrm{~K} \rightarrow 65 \mathrm{~K})$ and $60 \mathrm{~K}(260 \mathrm{~K} \rightarrow 200 \mathrm{~K})$, respectively, at $c=0.078$ $\left(T_{\text {solvus }}=1400 \mathrm{~K}\right)$ and $0.12\left(T_{\text {solvus }}=1600 \mathrm{~K}\right)$. However, the high discrepancy (overestimation) between the calculated and experimental results still remains in the high $\mathrm{Ru}-$ concentrations ( $c=0.078 \sim 0.12$ ). Thus, it is concluded that the results obtained by using the equilibrium lattice parameters $(a(T, c))$ of $\mathrm{Pd}_{1-c} \mathrm{Ru}_{c}$ may almost be unchanged from all of the above-mentioned results obtained by using the lattice parameters $(a(T, 0))$ of $\mathrm{Pd}$.

\section{Summary and Future Problem}

We presented the ab-initio calculations for the $T_{\text {solvus }}$ of the $\operatorname{Pd}_{1-c} \operatorname{Ru}_{c}(0.03 \leq c \leq 0.12)$ alloys, in which the $\mathrm{Ru}$ atoms are treated as impurities in Pd. The internal energies in the free energies are calculated by the present RSCE with the impurity IEs up to the 4-body, all of which are uniquely and accurately determined by the FPKKR calculations for perfect and impurity systems. ${ }^{9-12,14)}$ The configurational entropy calculations are based on the CVMT and CVMTO.

We firstly showed that the 2-body interaction is long-

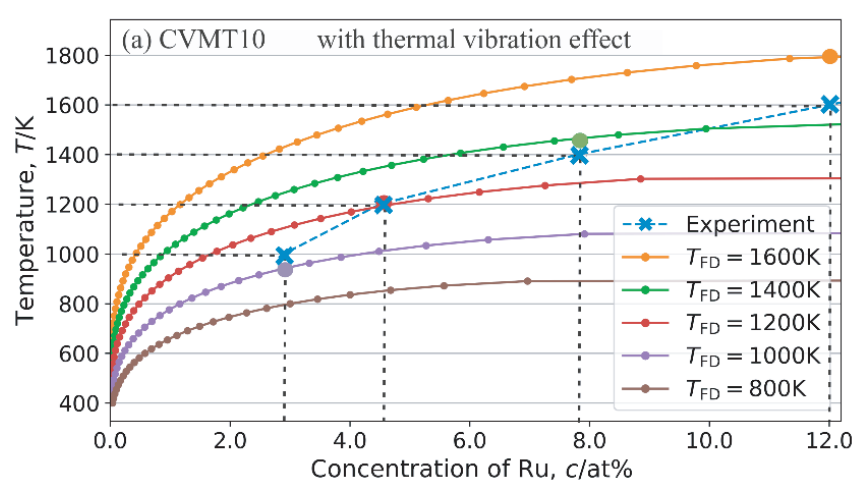

Fig. 6 Concentration dependence of the $T_{\text {solvus }}$ of the $\operatorname{Pd}_{1-c} \operatorname{Ru}_{c}(0.3 \leq c \leq$ 0.12) alloys, obtained by the CVMT10 calculations. The Ruconcentration is self-consistently determined by using lattice parameters $(a(T, c))$ of $\mathrm{Pd}_{1-c} \mathrm{Ru}_{c}$. See the text for details. 
range, and comparatively repulsive at the 2nd-, 4th-, and 9th-nns against the strong attraction at the 1st- $n n$. We also found that the following three effects attractively work on the IEs of the Ru impurities in Pd: (1) the local lattice distortion for the 1st- $n n$ Ru-Ru IE, (2) the lattice softening due to the thermal vibration at high temperatures, and (3) the electron excitation due to the FD distribution, being usually neglected in the ab-initio calculations. During the solvus temperature calculations, we realized that these three effects are very effective in reproducing the observed $T_{\text {solvus }}$.

We secondly showed the $T_{\text {solvus }}$ of the $\operatorname{Pd}_{1-c} \mathrm{Ru}_{c}(0.03 \leq$ $c \leq 0.12$ ) alloys by the free energy calculations based on the CVM. We found that the results obtained by the CVMT10 calculations, in which the $1 \mathrm{st}-n n$ IE is renormalized by including the $2 \sim 10$ th- $n n$ IEs, agree fairly well (within the error of $\sim 50 \mathrm{~K}$ ) with the observed $T_{\text {solvus }}$ of the $\mathrm{Pd}_{1-c} \mathrm{Ru}_{c}$ alloys, for the low Ru-concentrations up to $\sim 6 \%$, while the discrepancy (overestimation) between the calculated and experimental results becomes high for the high $\mathrm{Ru}-$ concentrations $(8 \sim 12 \%)$. We also found that the CVMTO10 calculations, in which the 1st- and 2nd-nn IEs can be taken into account, may correct the discrepancy in the high $\mathrm{Ru}$ concentration $(8 \sim 12 \%)$, if the 2 nd- $n n$ IE is suitably renormalized by including the 4 th- $n n$ IE.

Thus, we believe that the CVM calculations with the larger basic cluster such as the $\sim 13$ atom clusters, ${ }^{28)}$ at least exactly treating the IEs up to the repulsive 4th- $n n$ IE, can reproduce very well the observed $T_{\text {solvus }}$ of the $\mathrm{Pd}_{1-c} \mathrm{Ru}_{c}$ alloys, even for the high Ru-concentrations up to $\sim 12 \%$. The CVM calculations with the larger basic cluster including up to the 4th- $n n$ IE is strongly claimed to quantitatively determine the phase diagrams such as the concentration dependence of the observed $T_{\text {solvus }}$ of the long-range interaction systems.

\section{Acknowledgement}

The authors are grateful for the financial support from the Ministry of the Education, Culture, Science and Technology (JSPS KAKENHI Grant Numbers, 15K06422 and 16K06710). This work was partly supported by JSPS KAKENHI Grant Number JP15K14103 and by the Structural Materials for Innovation of the Cross ministerial Strategic Innovation Promotion Program (SIP) of Japan Science and Technology (JST). One of the authors (TM) appreciates their supports. Most of this study was completed when T.Hoshino was at the Institute for Material Research, Tohoku University. C. Liu and T. Hoshino would like to express their sincere thanks to the crew of the Center for Computational Materials Science of the Institute for Materials Research, Tohoku University, for their continuous support of the SR16000 supercomputing facilities.

\section{Appendix}

Appendix 1: Lattice parameter dependence of total energy per basic cluster of each phase consisting of the basic cluster used in the CVM calculations

In order to calculate the thermal vibration free energy using the Debye-Grüneisen model, ${ }^{1)}$ we must prepare some potential surfaces for the thermal vibration, that is, the lattice parameter dependences of the total energy per basic cluster, of each phase consisting of the basic cluster used in the CVM calculations. For the CVMT calculations, there are five kinds of basic clusters, as shown in Fig. 2 in Ref. 4) $(\mathrm{H}=\mathrm{Pd}, \mathrm{I}=\mathrm{Ru})$, where four atoms $\left(\mathrm{Pd}_{4-n} \mathrm{Ru}_{n}, n=0 \sim 4\right)$ are located at the 1st-nn tetrahedral sites. We show how to estimate the total energy $E_{\mathrm{Pd}_{4-n} \mathrm{Ru}_{n}}(a)$ per basic cluster of each phase consisting of the basic cluster $\mathrm{Pd}_{4-n} \mathrm{Ru}_{n}$ depending on the lattice parameter $(a)$. We can simply calculate $E_{\mathrm{Pd}_{4-n} \mathrm{Ru}_{n}}(a)$ as follows:

$$
E_{\mathrm{Pd}_{4-n} \mathrm{Ru}_{n}}(a)=4 \times E_{\mathrm{Pd}}(a)+\Delta E_{\mathrm{Ru}_{n} \text { in } \mathrm{Pd}}(a)
$$

where $E_{\mathrm{Pd}}(a)$ is the total energy of the Pd host per atom and $\Delta E_{\mathrm{Ru}_{n} \text { in } \mathrm{Pd}}(a)$ is the total energy change due to the presence of the $\mathrm{Ru}_{n}$ impurities in $\mathrm{Pd}$, all of which are obtained by the present ab-initio calculations. Of course, the interaction among the $\mathrm{Ru}$ impurities is restricted within a basic cluster, and no interaction among an infinite number of $\mathrm{Ru}_{n}$ in the separate basic clusters is included in eq. (A.1). However, if the equilibrium lattice parameter and the lattice parameter dependence of $E$ obtained by eq. (A.1) agree with those in the dense cluster system, where an infinite number of basic clusters $\left(\mathrm{Pd}_{4-n} \mathrm{Ru}_{n}\right)$ are arranged over the whole space, the Debye-Grüneisen model may be applicable for the dilute limit calculations presented here.

In order to give validity to the present approach, we compared the present results with the calculated results obtained from the periodic system of the basic $\operatorname{Pd}_{4-n} \mathrm{Ru}_{n}(n=0 \sim 4)$ clusters in the fcc unit cell. We noted in Fig. A.1 that the lattice parameter dependence of $E$ (including the value of the equilibrium lattice parameter of the total energy minimum), obtained by the present approach, agrees very well with those obtained by the periodic band calculations for each basic cluster. It is noted that all the energy minima are shifted to zero because the absolute value of the energy has no effect on the thermal vibration. These results show that the contribution from the interaction among $\mathrm{Ru}_{n}$ in the arrayed basic clusters, being ignored in the present approach, may be not important for the lattice parameter dependence of $E_{\mathrm{Pd}_{4-n} \mathrm{Ru}_{n}}(a)$. As a result, we may conclude that the important effect for the lattice parameter dependence of the total energy $E_{\mathrm{Pd}_{4-n} \mathrm{Ru}_{n}}(a)$ may be estimated by the present approach.

We now discuss how to treat the thermal vibration effect for the long-range 2-body IEs. The $m$-th $n n$ IE is written as follows:

$$
E_{m}^{2-\text { body }}(a)=\Delta E_{\mathrm{Ru}_{2} \text { in Pd }}^{m \text {-th neighbor }}(a)-2 \times \Delta E_{\mathrm{Ru}_{1} \text { in Pd }}(a)
$$

where $\Delta E_{\mathrm{Ru}_{2} \text { in Pd }}^{m \text {-th neighor }}(a)$ is the total energy change due to the presence of the $m$-th $n n \mathrm{Ru}-\mathrm{Ru}$ impurity pair in $\mathrm{Pd}$. We renormalized $\Delta E_{\mathrm{Ru}_{2} \text { in Pd }}(a)$ in eq. (A.1) by including these long-range 2-body IEs up to the 10th- $n n$. Using the resultant $E_{\mathrm{Pd}_{2} \mathrm{Ru}_{2}}(a)$, we calculated the thermal vibration free energy using the Debye-Grüneisen model discussed in Sect. 2.2. Thus, within the CVMT approximation, we can take into account the thermal vibration effect for the long-range 2-body IEs. 


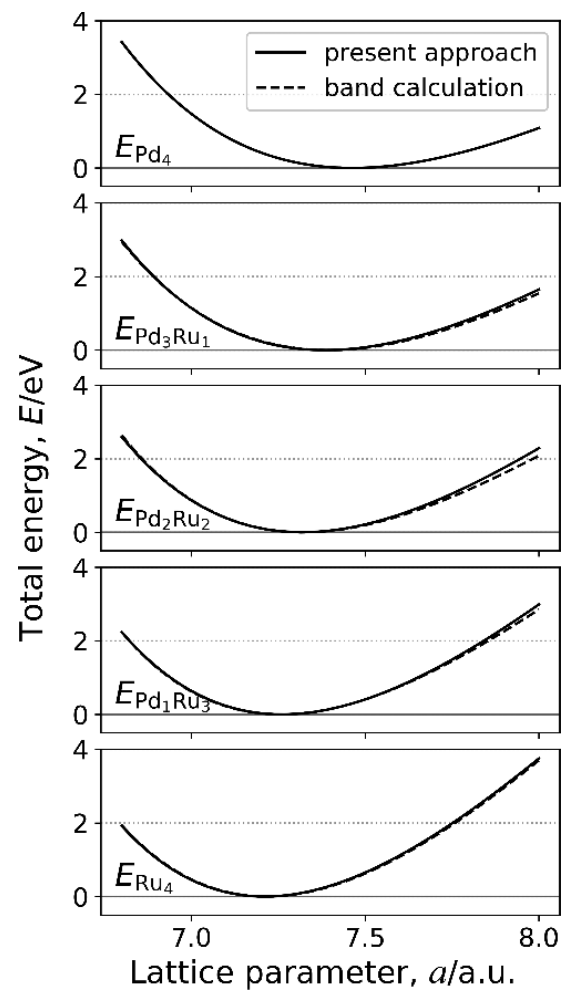

Fig. A.1 Lattice parameter dependence of the total energies $\left(E_{\mathrm{Pd}_{4-n} \mathrm{Ru}_{n}}\right)$ per basic clusters $\left(\mathrm{Pd}_{4-n} \mathrm{Ru}_{n}, n=0 \sim 4\right)$ used in the CVMT approximation obtained by the present approach. The calculated results by the band calculations with the unit cells $\left(\mathrm{Pd}_{4-n} \mathrm{Ru}_{n}, n=0 \sim 4\right)$ are also shown for comparison. All these calculations were executed with the FD distribution at $T_{\mathrm{FD}}=800 \mathrm{~K}$. There is almost no difference between the calculated results obtained by the two approaches. See the text for details.

\section{Appendix 2: Calculation procedure for temperature de- pendence of solid solubility limit}

In order to calculate the configurational entropy in the disordered state (in eq. (5)) at a given temperature, we used the cluster variation method within the tetrahedron approximation (CVMT) introduced by Kikuchi. ${ }^{29)}$ Kikuchi also designed the natural iteration (NI) technique for the minimization of the grand potential of the disordered state. As shown in Ref. 29), the grand potential per atom is written in the following form:

$$
\Omega=F_{\mathrm{dis}}-\sum_{i} \mu_{i} \times x_{i}
$$

where $F_{\text {dis }}$ is the free energy of the disordered state, and $\mu_{i}$ and $x_{i}$ are the chemical potential and concentration of the $i$-element (here, $i=\mathrm{Pd}$ or $\mathrm{Ru}$ ), respectively. It is noted that $\Omega$ is the function of cluster probabilities $\left\{\omega_{i j k l}\right\}$, temperature $(T)$ and chemical potentials $\left\{\mu_{i}\right\}$, as shown in Ref. 29).

In order to minimize the free energy difference, $\Delta F$ (eq. 5), we can use this formalism by regarding the 2 nd term in eq. (A.3) as the internal energy of the segregation state, which is written as,

$$
E_{\mathrm{seg}}=2 \times\left(E_{\mathrm{Pd}_{4}} \times x_{\mathrm{Pd}}+E_{\mathrm{Ru}_{4}} \times x_{\mathrm{Ru}}\right)
$$

where $E_{\mathrm{Pd}_{4}}\left(E_{\mathrm{Ru}_{4}}\right)$ is the total energy of the tetrahedron cluster $\mathrm{Pd}_{4}\left(\mathrm{Ru}_{4}\right)$ in the Pd host, discussed in Sect. 2.1. and factor 2 means that the $\mathrm{N}$-lattice points constitute $2 \times \mathrm{N}$ tetrahedrons in the fcc structure. As a result, it is shown that $\Omega$ equals $\Delta F$ (eq. 5) as follows:

$$
\begin{aligned}
\Omega & =F_{\text {dis }}-\sum \mu_{i} \times x_{i} \\
& =F_{\text {dis }}-2 \times\left(E_{\mathrm{Pd}_{4}} \times x_{\mathrm{Pd}}+E_{\mathrm{Ru}_{4}} \times x_{\mathrm{Ru}}\right) \\
& =\Delta F
\end{aligned}
$$

It is noted that $\mu_{i}(i=\mathrm{Pd}$ or $\mathrm{Ru})$ is replaced by $2 \times E_{i i i i}$. Equation (A.5) may also be transformed as follows,

$$
\begin{aligned}
\Omega= & F_{\text {dis }}-\left(E_{\mathrm{Pd}_{4}}+E_{\mathrm{Ru}_{4}}\right) \times\left(x_{\mathrm{Pd}}+x_{\mathrm{Ru}}\right) \\
& -\left(E_{\mathrm{Pd}_{4}}-E_{\mathrm{Ru}_{4}}\right) \times\left(x_{\mathrm{Pd}}-x_{\mathrm{Ru}}\right) \\
= & F_{\text {dis }}-\left(E_{\mathrm{Pd}_{4}}+E_{\mathrm{Ru}_{4}}\right)-\left(E_{\mathrm{Pd}_{4}}-E_{\mathrm{Ru}_{4}}\right) \times\left(x_{\mathrm{Pd}}-x_{\mathrm{Ru}}\right)
\end{aligned}
$$

where we used the relation $x_{\mathrm{Pd}}+x_{\mathrm{Ru}}=1$. Since the origin of the energy can be changed, we used the origin of eq. (A.6) to satisfy the following relation

$$
E_{\mathrm{Pd}_{4}}+E_{\mathrm{Ru}_{4}}=0
$$

Then, eq. (A.6) is transformed into

$$
\begin{aligned}
\tilde{\Omega} & =F_{\mathrm{dis}}-\left(E_{\mathrm{Pd}_{4}}-E_{\mathrm{Ru}_{4}}\right) \times\left(x_{\mathrm{Pd}}-x_{\mathrm{Ru}}\right) \\
& =F_{\mathrm{dis}}-\left(\tilde{\mu}_{\mathrm{Pd}} \times x_{\mathrm{Pd}}+\tilde{\mu}_{\mathrm{Ru}} \times x_{\mathrm{Ru}}\right)
\end{aligned}
$$

where $\tilde{\mu}_{\mathrm{Pd}}=-\tilde{\mu}_{\mathrm{Ru}}=E_{\mathrm{Pd}_{4}}-E_{\mathrm{Ru}_{4}}$. It is noted that the final formula (eq. (A.8)) is a function of the cluster probabilities $\left\{\omega_{i j k l}\right\}$ and temperature $T$. Thus, using the NI technique along with the total energies $\left\{E_{i j k l}\right\}$ of the tetrahedron clusters in the $\mathrm{Pd}$ host, we can easily obtain $\left\{\omega_{i j k l}\right\}$ by minimizing eq. (A.8) with respect to $\left\{\omega_{i j k l}\right\}$ at a given temperature $T$.

\section{REFERENCES}

1) T. Mohri and Y. Chen: J. Alloy. Compd. 383 (2004) 23-31.

2) T. Mohri, T. Morita, N. Kiyokane and H. Ishi: J. Phase Equilib. 30 (2009) 553-558

3) J.W. Connolly and A.R. Williams: Phys. Rev. B 27 (1983) 5169.

4) M. Asato, T. Mizuno, T. Hoshino and H. Sawada: Mater. Trans. 42 (2001) 2216-2224

5) T. B. Massalski, H. Okamoto, P. R. Subramanian and L. Kacprazak: Binary Alloys Phase Diagrams, 2nd. ed. (ASM International, New York, 1990)

6) By using the FPKKR calculations, we also studied the distance dependence, up to the 20th- $n n$, of the 2-body IEs of the Rh impurities in Pd and the local lattice distortion effect for the 1st-nn 2-body IE. We found: (1) the 2-body interaction is weakly repulsive at the 2nd-, 4th-, and 9th-nns against the strong attraction at the 1st-nn. (2) the local lattice distortion effect for the $1 \mathrm{st}-n n$ IE is weakly attractive $(\sim-0.01 \mathrm{eV})$, compared to that $(\sim-0.03 \mathrm{eV})$ for the $\mathrm{Ru}$ impurities in Pd. By the free energy calculations based on the CVMT, taking into account these IEs together with the lattice thermal vibration effect, we can reproduce very well the observed $T_{\text {solvus }}$ of the $\mathrm{Pd}_{1-c} \mathrm{Rh}_{c}(0.09<$ $\mathrm{c}<0.12$ ) alloys. These results will be published in a subsequent paper (C. Liu et al., in preparation).

7) T. Hoshino, M. Asato, S. Tanaka, F. Nakamura and N. Fujima: Intermetallics 14 (2006) 913-916.

8) C. Liu, M. Asato, N. Fujima and T. Hoshino: Mater. Trans. 54 (2013) 1667-1672.

9) T. Hoshino, M. Asato, R. Zeller and P. H. Dederichs: Phys. Rev. B 70 (2004) 094118-1-7.

10) F. Nakamura, T. Hoshino, S. Tanaka, K. Hirose, S. Hirosawa and T. Sato: Trans. Mater. Res. Soc. Jpn. 30 (2005) 873-876.

11) M. Asato, H. Takahashi, T. Inagaki, N. Fujima, R. Tamura and T. Hoshino: Mater. Trans. 48 (2007) 1711-1716.

12) T. Hoshino, M. Asato and N. Fujima: J. Alloy. Compd. 504 (2010) S534-S537.

13) For a review, see A. Gonis, M. Slutier, P.E.A. Turchi, G.M. Stocks and D.M. Nicholson: J. Less Common Met. 168 (1991) 127-144.

14) C. Liu et al., submitted to Mater. Trans. 
15) M. Asato, A. Settles, T. Hoshino, T. Asada, S. Blugel, R. Zeller and P.H. Dederichs: Phys. Rev. B 60 (1999) 5202-5210.

16) T. Hoshino, M. Asato, T. Mizuno and H. Fukushima: Mater. Trans. 42 (2001) 2206-2215.

17) R. Zeller: Phys. Rev. B 55 (1997) 9400-9408.

18) R. Zeller, M. Asato, T. Hoshino, J. Zalbloudil, P. Weinberger and P.H. Dederichs: Philos. Mag. B 78 (1998) 417-422.

19) T. Hoshino, W. Schweika, R. Zeller and P.H. Dederichs: Phys. Rev. B 47 (1993) 5106-5117.

20) K. Wildberger, P. Lang, R. Zeller and P.H. Dederichs: Phys. Rev. B 52 (1995) 11502-11508

21) N. Papanikolaou, R. Zeller, P.H. Dederichs and N. Stefano: Phys. Rev. B 55 (1997) 4157-4167.

22) T. Hoshino, N. Papanikolaou, R. Zeller, P.H. Dederichs, M. Asato and N. Stefanou: Comput. Mater. Sci. 14 (1999) 56-61.

23) M. Asato, C. Liu, K. Kawakami, N. Fujima and T. Hoshino: Mater. Trans. 55 (2014) 1248-1256.

24) C. Liu, M. Asato, N. Fujima and T. Hoshino: Phys. Procedia 75 (2015) 1088-1095.

25) The density of states (DOS) of 4d-element (and 3d-element) metals are considerably high at the Fermi levels because of the narrow 4d-bands existing around the Fermi levels, as shown in the textbook (Calculated Electronic Properties of Metals, by V. L. Morruzi, J. F. Janak, and A. R. Williams, Pergamon Press Inc, 1978). The spin polarization energies of $4 \mathrm{~d}$-adsorbate atoms $(\mathrm{Nb} \sim \mathrm{Ru})$ on a $\mathrm{Ag}(001)$ surface, also being the systems with the high DOS at the Fermi level, are largely reduced with the increasing $T_{\mathrm{FD}}$, as shown in Ref. 20). Thus, we expect that the $T_{\mathrm{FD}}$-dependence of I-I ( $\mathrm{I}=4 \mathrm{~d}$ elements) IEs in Pd may also become large. We are now executing the systematic calculations for the $T_{\mathrm{FD}}$-dependence for I-I (I $=4 \mathrm{~d}$ elements) IEs in Pd. We have already found that the $\mathrm{Rh}-\mathrm{Rh}$ repulsion at the $2 \mathrm{nd}-n n$ is considerably reduced with the increasing $T_{\mathrm{FD}}$ (from $0.0093 \mathrm{eV}$ at $200 \mathrm{~K}$ to $0.003 \mathrm{eV}$ at $900 \mathrm{~K}$ ). These results will be published in a subsequent paper (C. Liu et al., in preparation).

26) W. Schweika: Structural and Phase Stability of Alloys, ed. by J. L. Möran-Löpez et al., (Plenum Press, New York 1992) pp. 53-64.

27) W. Schweika: Disordered Alloys, (Springer Tracts in Modern Physics 141, Springer 1998) pp.51-54.

28) D. de. Fontaine: Solid State Physics, Vol.47, ed. by H. Eherenreich and D. Turnbull, (Academic Press, London 1994) pp.33-176.

29) R. Kikuchi: National Bureau of Standards SP-496 (1977) 967-998. 